\title{
GALACTIC BACKGROUND SURVEYS AND THE GALACTIC HALO
}

\author{
J. E. BALDWIN \\ Mullard Radio Astronomy Observatory, Cavendish Laboratory \\ Cambridge, England
}

Two surveys of the galactic background radiation at low frequencies have recently been made at Cambridge. At $38 \mathrm{Mc} / \mathrm{s}$, a survey of the sky between declinations $-20^{\circ}$ and $+70^{\circ}$ has been made by Blythe [1] using a new type of pencil-beam aerial system. This instrument, consisting of an array 1200 feet long in an east-west direction together with a small movable aerial, used the principle of aperture synthesis to provide a beam $2.2 \times 2.3$ at the zenith increasing to $2: 2 \times 7.4$ at an angle of 70 degrees from the zenith.

The map of this survey shows no trough of absorption along the galactic equator, indicating that the effects of ionized hydrogen in the galactic plane are not very important at this frequency. This result confirms Shain's observations of absorption at $19.7 \mathrm{Mc} / \mathrm{s}$ [2].

Several nearby $\mathrm{H}$ II regions show in absorption against the galactic background, which enables estimates to be made of their emission measures. Some eighty radio sources were observed in the survey, of which many of the extended ones showed a strong concentration toward the galactic equator. The map also shows a large amount of detail in the extraordinary belt of emission extending from the galactic plane near $l=0^{\circ}$ toward the north galactic pole.

In some directions away from the galactic plane the survey had inadequate sensitivity to show the details of the background and, in regions close to the bright sources, the observations were unreliable due to the existence of side lobes of the main beam. A survey is now in progress using a much larger instrument working on the same principle, which will give a map of the sky at $38 \mathrm{Mc} / \mathrm{s}$ with a resolving power of $1 \times 1$ degree. The sensitivity, phase stability, and primary resolving power of the individual parts of the aerial system have been considerably improved compared with that described above. The fixed portion of the aerial has an extent of 3200 by 40 feet and the movable portion 100 by 40 feet.

At $160 \mathrm{Mc} / \mathrm{s}$ one section of the radio telescope used in the $3 \mathrm{C}$ survey of radio stars has been used for a survey of the background radiation. The beamwidth of the aerial was 1.2 in right ascension and 7 degrees in declination to halfpower points. A modified type of Dicke switching system was used in the observations. Since large areas of the sky have brightness temperatures close to room temperature at $160 \mathrm{Mc} / \mathrm{s}$, measurements accurate to about one per cent of the total background could be made. The zero level of temperature 
is known to within approximately $\pm 8^{\circ} \mathrm{K}$. Observations of about one-half of the sky visible from Cambridge have been reduced so far, and preliminary results for the portion of sky between right ascensions $4^{\mathrm{h}}$ and $10^{\mathrm{h}} 30^{\mathrm{m}}$ are shown in Fig. 1. The contour interval is approximately $8^{\circ} \mathrm{K}$. The half-power extent of the beam is indicated by the solid black region surrounding the Crab nebula. The accurate temperatures of this survey, particularly in regions away from the galactic plane, may be compared with those obtained from the same aerial structure at $81.5 \mathrm{Mc} / \mathrm{s}$ [3] to deduce the spectrum of the background radiation in different directions. In this way it may be possible to distinguish between the radiation from the galactic halo and that from extragalactic sources.

The physical conditions in the galactic halo are of particular interest at the present time in connection with theories of the production of radio emission. Observations by Reber and Ellis [4] in Tasmania at a frequency of $2.1 \mathrm{Mc} / \mathrm{s}$ provide useful data for estimating the possible value of the interstellar gas density in the galactic halo. Since the tenuous gas at heights above the galactic plane greater than about 500 parsecs is likely to be largely ionized, the absorption coefficient for very low-frequency radio waves may be appreciable. A comparison of the angular distribution of radio emission at $2.1 \mathrm{Mc} / \mathrm{s}$ with that observed at frequencies close to $100 \mathrm{Mc} / \mathrm{s}$ has been made (Baldwin [5]) showing that the optical depth for free-free emission in the interstellar gas is less than 0.5 through the depth of the galactic halo, about 10,000 parsecs.

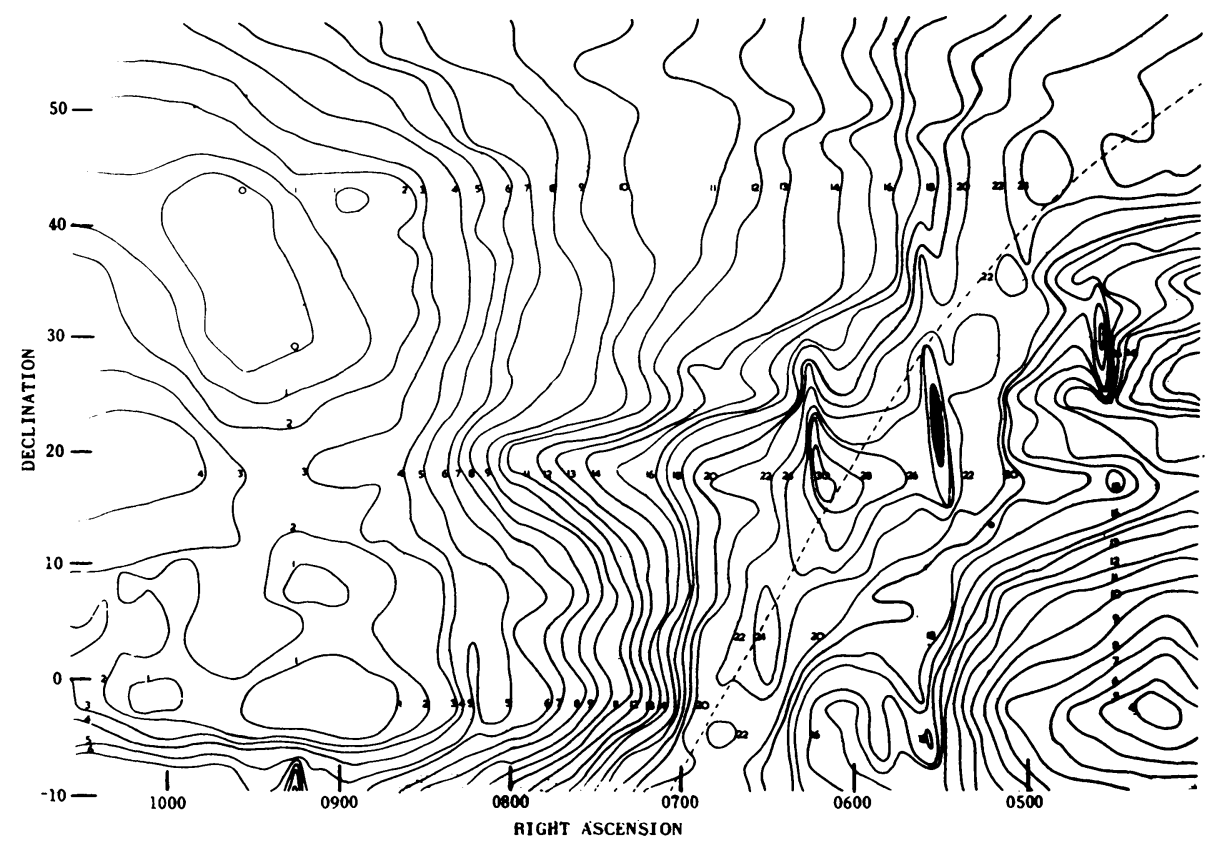

FIG. 1. Coutours of radio brightness at $160 \mathrm{Mc} / \mathrm{s}$. One unit is equivalent to $8^{\circ} \mathrm{K}$. 
If the kinetic temperature of the gas is $10,000^{\circ} \mathrm{K}$, as it is in the nearby $\mathrm{H} \mathrm{II}$ regions, the mean gas density in the halo must be less than $2 \times 10^{-2} \mathrm{~cm}^{-3}$. The analysis of very low frequency radio observations can thus provide a much lower limit to the gas density in the galactic halo than can be obtained in other ways.

\section{REFERENCES}

[1] Blythe, J. H. M.N.R.A.S. 117, 652, 1957.

[2] Shain, C. A. Aust. J. Phys. 10, 195, 1957.

[3] Baldwin, J. E. M.N.R.A.S. 115, 684, 1955.

[4] Reber, G., and Ellis, G. R. J. Geophys. Res. 61, 1, 1956.

[5] Baldwin, J. E. The Observatory, 78, 166, 1958.

\section{Discussion}

Westerhout: In Australia, C. M. Wade and J. V. Hindman are making a survey at $1393 \mathrm{Mc} / \mathrm{s}$ with a 1.4-degree beamwidth. The results of this survey will be very interesting in comparison with the northern hemisphere results. In particular the maximum in the density at 3.5 kiloparsecs needs confirmation by southern observations before we can really assume circular symmetry and try to find relationships between this maximum and the expanding region inside 3 kiloparsecs.

van de Hulst: Is there a discrepancy between the distribution of volume emissivity in the halo presented now by Mills and that presented some years ago by Baldwin? Baldwin could fit to his data a model in which the emissivity is homogeneous all the way to 15 kiloparsecs, whereas Mills found the emissivity to drop sharply just beyond the sun.

F. G. Smith: Mills's analysis of the distribution far from the plane has led to a model of the halo very similar to that published by Baldwin. The total emission is much the same, except that Baldwin gave two values depending on the extent of the halo. The question I ask concerns primarily the extent of the outer parts. Baldwin found the total emission and the ellipticity to depend on the extragalactic component. What value did Mills use for this component?

Mills: The value assumed was $250^{\circ} \mathrm{K}$ or about one-third of the minimum observed sky temperature. This was the most probable value derived from Shain's results. However, Shain suggests limits of one-half to one-tenth. These would affect the distribution obtained for the outer part substantially, but not the ellipticity, which was based on the distribution inside the solar distance. I have not yet computed the effect on the total emission.

Baldwin: Mills has assumed a value of $250^{\circ} \mathrm{K}$ for the extragalactic component. The model of the galactic halo given in M.N.R.A.S. 115, 690, 1955 corresponding to this has a semimajor axis of about 13 kiloparsecs. This value agrees quite well with that derived by Mills.

Hoyle: Does the spiral-arm effect mentioned by Mills require more direc- 
tivity of emission along the spiral arms than would be given by a large number of isotropic small sources within the arms?

Mills: No, the calculation of the equivalent radial distribution was based on the assumption of isotropic radiators. The fact that the arms were so clearly delineated suggests that this assumption was correct.

Brown: How do the models of the galactic corona proposed by either Mills or Baldwin agree with the corona which is found around the Andromeda nebula, for example, by the Dutch?

Mills: The Andromeda nebula is given by Seeger as an oblate spheroid of axial ratio about $2: 1$ and diameter to half-emissivity points of 50 kiloparsecs. We find an axial ratio of $1.5: 1$ and a diameter of 20 kiloparsecs. The total emission, however, appears comparable.

van de Hulst: Baldwin implied that by measuring the background at different frequencies it would in due time be possible to distinguish the extragalactic from the halo component. Does this not require the assumption that the spectrum is identical throughout the halo, and is this assumption plausible?

Baldwin: If it is found that all the small features of the galactic halo at high latitudes have the same spectrum, it is reasonable to suppose that the spectrum of the galactic halo is constant throughout its depth. If the extragalactic component has a different spectrum from that of the halo it should be possible to separate these components by making observations at different frequencies.

Oort: Mills has mentioned the problem of the influence of extragalactic radiation on the irregularities in the total radiation observed in high latitudes. The available data (in particular those on the Virgo and Coma clusters, where very dense concentrations give little or no radiation) do not appear to lend support to the idea that much of the irregularity observed could be extragalactic in origin. And it was hardly to be expected that this could be so. The volume of space covered by the Shapley-Ames catalog may be considered to be representative for the average of the universe as far as small density is concerned. The average expansion shown by these nebulae is of the order of $1,000 \mathrm{~km} / \mathrm{second}$. An estimate of the radiation to be expected from the rest of the universe then gives a minimum of 200 times that from the volume covered by the Shapley-Ames catalog. With the surface density in the Virgo cluster about 10 times the average of the Shapley-Ames nebulae above 30 degrees latitude, the fluctuation caused by the cluster would not be expected to be more than 5 per cent of the total extragalactic background, or perhaps 2 per cent of the radiation from galactic corona and extragalactic background together. This is entirely negligible compared to the variations observed in high latitudes, and seems to indicate that these latter must be phenomena of the galactic corona.

Mills: Our observations appear to suggest that many dense clusters radiate considerably more than expected from their component galaxies. It is not unreasonable to suppose that this should happen; a very dense concentration of galaxies seems likely to produce strong continuum radiation because of the 
high gas velocities involved. In fact a "radio cluster" analogous to a " radio galaxy" is not inconceivable. Until more work can be done it appears best to keep an open mind on this question.

Oort: The Virgo cluster does not show any effect. This indicates that the contribution by irregularities in extragalactic distribution is probably small.

Pecker: What are the optical depths at different wavelengths and at different points of the surveys? Is it necessary to take possible high optical depths into account in order to derive densities (or are the self-absorption effects of some importance)?

Westerhout: The optical depth of the ionized hydrogen in the region $l-l_{c}<30$ degrees on the galactic ridge is on the average around 0.001 at $1390 \mathrm{Mc} / \mathrm{s}, 0.2$ at $85 \mathrm{Mc} / \mathrm{s}$, and lower farther along the galactic ridge and at other latitudes.

Shakeshaft: In his consideration of the total expected extragalactic component has Professor Oort included the contribution from abnormal galaxies as well as from normal galaxies?

Oort: I did not consider the background due to the integrated radiation of distant radio sources (abnormal galaxies). This type of radiation should be practically isotropic, apart from the sources that are bright enough to be observed individually. The random fluctuations in this radiation would be quite different in character from the fairly large-scale variations observed in high latitudes. The general background area to abnormal galaxies would weaken still further the effects to be expected from nearby local groupings such as the Virgo cloud.

Brown: In his estimate of the upper limit to the irregularities in the isophotes to be expected from extragalactic nebulae Professor Oort based his figures on the Shapley-Ames galaxies. How has he taken into account the anisotropic angular distribution of these galaxies over the sky?

Oort: It is conceivable that dense groups of galaxies might emit more than would correspond to the increased number of galaxies because of some kind of interaction. The observations of Virgo and Coma, however, indicate that any effect of this sort, if it exists, must be small. 